\title{
Stoma Complications
}

Timuçin Erol *, [MD]
ORCID: 0000-0002-3475-3639

Hacettepe University, Faculty of Medicine, Department of General Surgery

Hacettepe University Hospital, Department of General

Surgery, 06230, Sinhıye, Ankara / Turkey

e- mail : timucinerol@hacettepe.edu.tr

Phone Number: +90 5323367645

Received: 10 March 2019, Accepted:26 April2019,

Published online: 30 June 2019

\section{ne ABSTRACT Cen}

An improper stoma creation can cause many complications, varying from minor to life-threatening ones. Conversely, a good functioning stoma, at the ideal site improves patient's quality of life. Most of the stoma complications occur in the early postoperative period and all clinicians must be familiar to these complications. All measures before and during operation must be taken to avoid these complications. Careful follow up after post-operative period can help early diagnosis, proper treatment quick recovery of the patient. This review focus on common complications and treatment options of stoma creation.

Key words: Stoma, ileostomy, colostomy

\section{INTRODUCTION}

Although stoma creation thought to be a relatively simple procedure, this procedure has high morbidity or even mortality rates [1,2]. Even after discharge from hospital, a poor functioning stoma affects the patient's quality of life negatively [3,4]. Stoma creation generally, is the last step of a complex abdominal surgery which performed either for a benign condition like inflammatory bowel diseases, diverticulitis, trauma or premalignant/malignant diseases like familial adenomatosis coli, colorectal cancer. It can be performed under elective or emergency conditions. Loop or end ostomy creation is possible which is decided according to the patient's condition, underlying disease or surgeon's preference. Even in the optimal circumstances, these complications can occur but every technical measure must be taken in order to create a good functioning stoma [5]. Marking of the proper stoma place before the operation is the first and crucial step of creating a good stoma. Multiple studies showed pre-operative marking of stoma site by a stoma therapist significantly decrease stoma complications $[6,7]$. During marking for the most suitable site, patient age, gender, body mass index, visual capacity, dexterity, presence of other stomas must be taken into consideration. Inspection while the patient is standing, bending and sitting, marking of a flat site away from prior incisions, bone protuberances and marking of a site underbelly line which patient can see, is the ideal location [8].

Stoma complications can be divided into two groups, early and late complications. Early complications occur within the first month of the operation and most of the complications occur in this period. Peristomal skin problems, ischemia, necrosis, retraction, infection-related problems, mucocutaneous separation are the most common early complications. Late complications present one month 
after the operation and most common late complications are parastomal hernia, stomal prolapse, and retraction/stenosis. Type of the stoma, creation of stoma for an emergency condition, body mass index and stoma length are the other factors contributing to these complications $[2,9]$.

Study of Malik et.al which they summarized 18 randomized clinical trials on stoma complications overall complication rates of loop ileostomy, loop colostomy and end colostomy are 14.3 percent (\% 2.962.2), 26.3 percent (\%13.9-100), 2.6 percent (\% 2.0100). Although the highest rate of complication presents in end colostomy group, mainly parastomal hernia, this group consists from patients whose colostomies performed after abdominoperineal resection for low rectal cancers, whose follow up period are relatively longer as these ostomies are permanent.

\section{Peristomal Skin Complications}

Peristomal skin complications are the most common early complication of stoma creation and mostly because of improper stoma site or bad appliance which results in leakage and skin irritation [10]. It can be quite high as 3 to 42 percent mainly after ileostomies [8]. Leakage and direct exposure small bowel ingredient which is high alkaline and caustic leads the skin irritation which requires local therapies. These treatments make the application of adhesives difficult and frequent change of ostomy applicators leads to more skin problems (Figure 1). Proper technique for the creation of stomas can decrease skin complications.

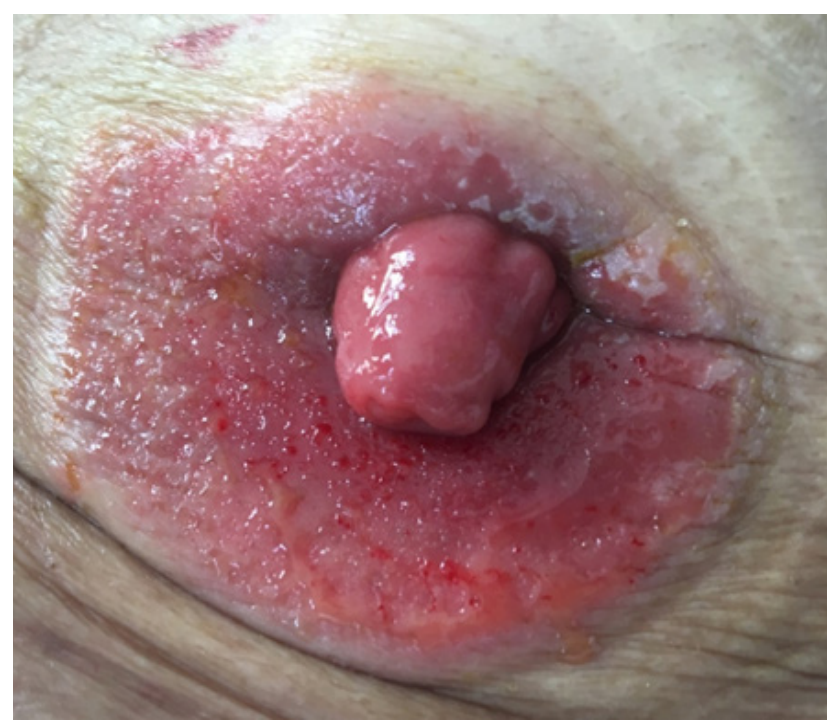

Figure 1. Peristomal skin irritation
Peristomal Candida Albicans infection is common because of the proper environment. A red rash with satellite lesions usually enough for the diagnosis. Once diagnosed antifungal treatment with miconazole nitrate $\% 2$ powder generally enough.

Pyoderma Gangrenosum is another rare condition which needs careful evaluation and treatment as approximately half of these patients have systemic diseases. Peristomal pyoderma gangrenosum generally associates with inflammatory bowel disease and activity of disease shows correspondence with symptoms [11]. Small erythematous pustules merge rapidly and form painful undermined, violaceous bordered ulcers. Diagnose made by excluding other reasons like malignancy and infection. Misdiagnose as contact dermatitis, skin irritation or infection is common. Once diagnosed topical therapies, intra-lesional corticosteroid injection, surgical debridement, systemic corticosteroid, anti-inflammatory or immunosuppressant medications can be used according to disease severity [12].

\section{Stomal Ischemia, Necrosis}

Ischemia due to venous obstruction or inadequate arterial supply because of mesenteric trimming or tension can be seen in 1-10 percent of colostomies and 1-5 percent of ileostomies [13]. Obesity and emergency stoma creation are the risk factors for ischemia. Degree of the ischemia and necrosis is important during management. Early venous congestion tends to resolve after the resolution of edema and regression of abdominal distention but arterial compromise can cause ischemia and necrosis gradually. Superficial ischemia or necrosis which did not progress under fascia can manage non operatively by close and carefully repetitive examinations (Figure 2).

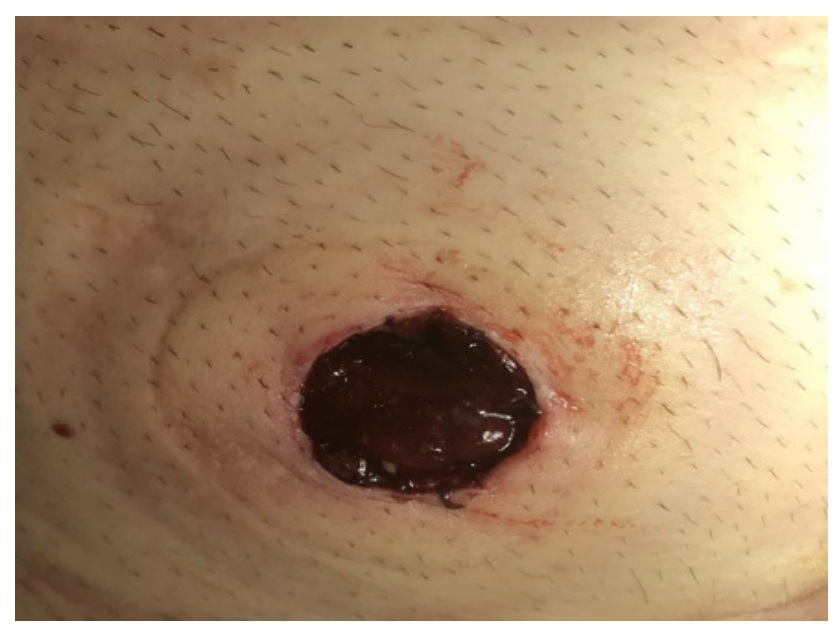

Figure 2. Stomal ischemia 
A surgeon must be aware that these stomas are prone to stenosis and retraction. On the other hand, early intervention is crucial in case of full thickness ischemia and necrosis beyond the fascia. Reresection of the ischemic segment of bowel and re-maturation or relocation of stomas are the treatments of choice.

\section{Stoma Retraction}

Stoma retraction is another complication which happened after 6 percent of colostomy and 3-17 percent of ileostomy formation (5). Insufficient bowel mobilization, ischemia, obesity, malnutrition, and immunosuppression are the main reasons for retraction. Tension on the bowel cause inversion of the mucocutaneous junction and stoma retraction generally with stenosis (Figure $3 a-b$ ).
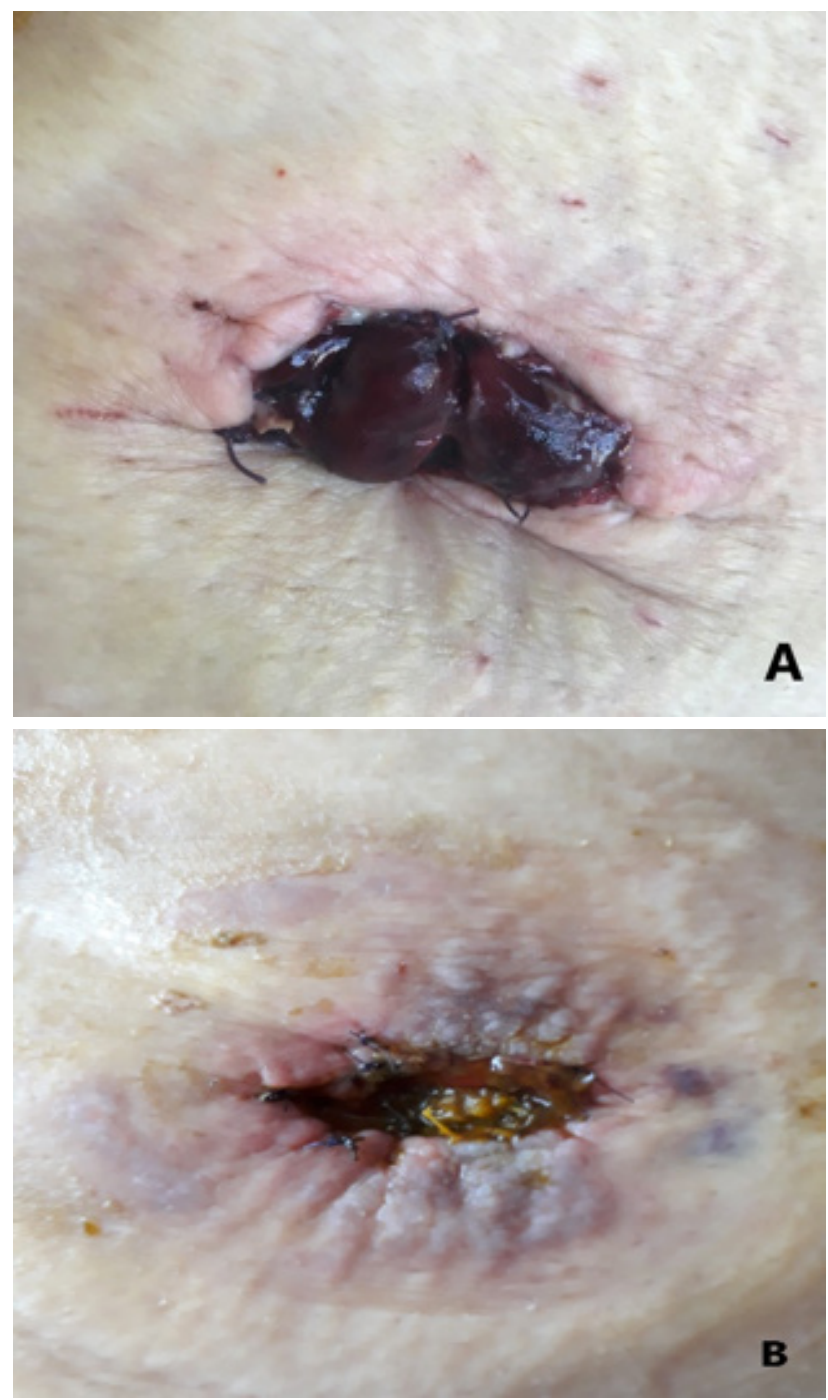

Figure 3 A-B. Early ischemia and gradually stenosis of colostomy

Cottom et.al found that the most significant complication within the 3 weeks of surgery is stoma retraction [14]. According to this study height of the stoma above skin either ileostomy or colostomy is directly correlated with stoma problems. Although local measures like using convex adaptors taken most of the time stoma reversal becomes inevitable.

\section{Stoma Prolapse}

Stoma prolapse defined as the protrusion of the bowel through ostomy. Rate of stomal prolapse varies from 3 to 26 percent depending on stoma type (loop or end), bowel used for a stoma (ileum or colon) and timing of operation (elective or emergent) [15]. Other risk factors are age, obesity, bowel obstruction during stoma creation and absence of preoperative marking of stoma site. Loop colostomies particularly efferent loop have higher prolapse rates [16]. Prolapse can cause mild to serious complications (Figure 4).

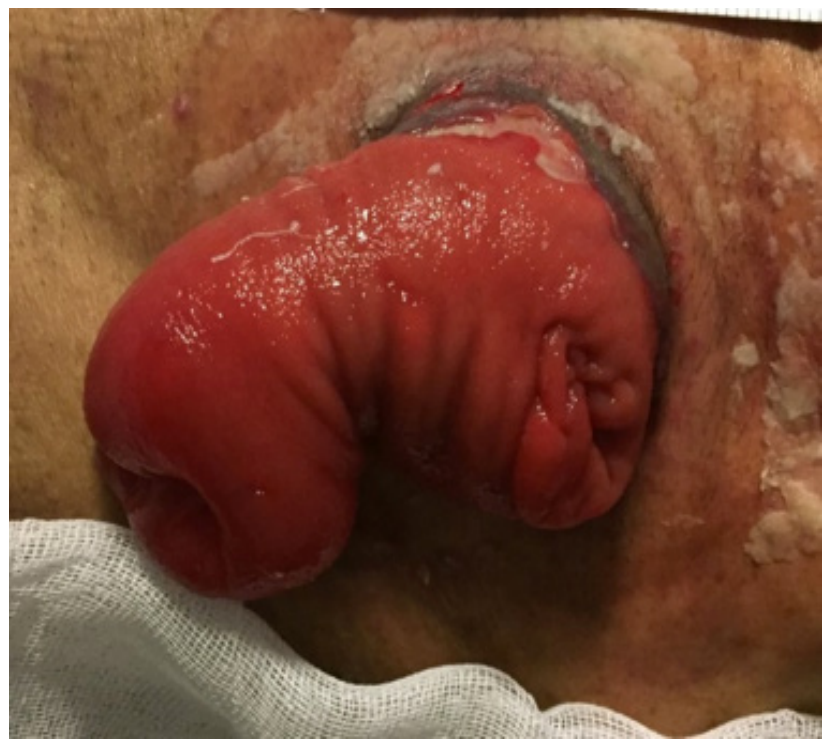

Figure 4. Stomal prolapsus

Skin irritation, poor fitting, pain, obstruction, bleeding, ischemia, and necrosis are common symptoms. Even asymptomatic patient can feel discomfort because of the vision of the prolapsed segment. Spontaneous reduction by gentle pressure on the prolapsed segment is generally possible (Figure 5). When edema present sugar application can relief the edema and helps reduction.

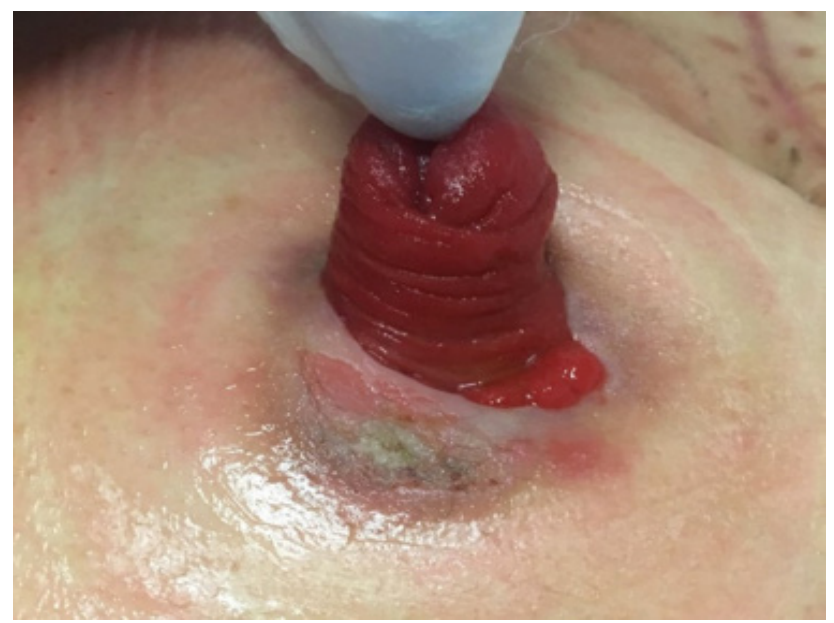

Figure 5. Stomal prolasus which reduced manually 
Presence of Ischemia and necrosis is a clear indication for emergency reoperation. In elective setting closure of the temporary stomas if possible is one of the treatment options. Relocation of the stoma during operation in case of poor fitting condition must be considered. Simple resection of the prolapsed segment from $1 \mathrm{~cm}$ above the mucocutaneous junction and re-anastomosis is generally performed operation and is generally enough and narrowing of skin aperture is recommended. Laparoscopic enteropexy is a new technique which reduces the prolapsed segment and fixes the bowel to the abdominal wall. But this technique needs further investigation [17]. In case of accompanying parastomal hernia, concomitant hernia repair must be considered.

\section{Parastomal Hernia}

Parastomal hernia is a late complication of stoma creation and can be defined as the protrusion of abdominal contents from the abdominal wall through defect created for stoma formation (Figure 6).

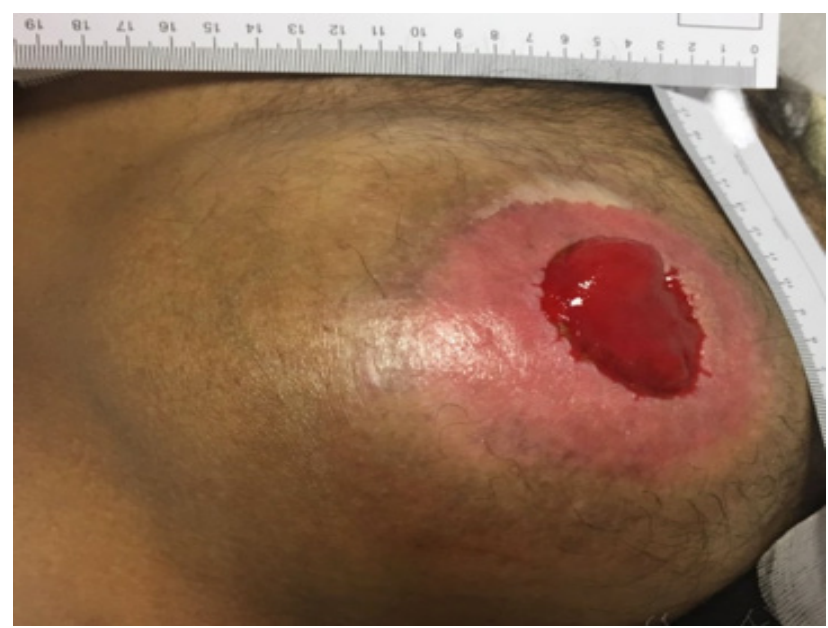

Figure 6. Parastomal hernia

Indeed, it's an incisional hernia related to abdominal wall stomas $[18,19]$. Although some of them are asymptomatic skin problems due to poor appliance, pain, obstructive symptoms, strangulation, and even perforation is possible (Figure 7

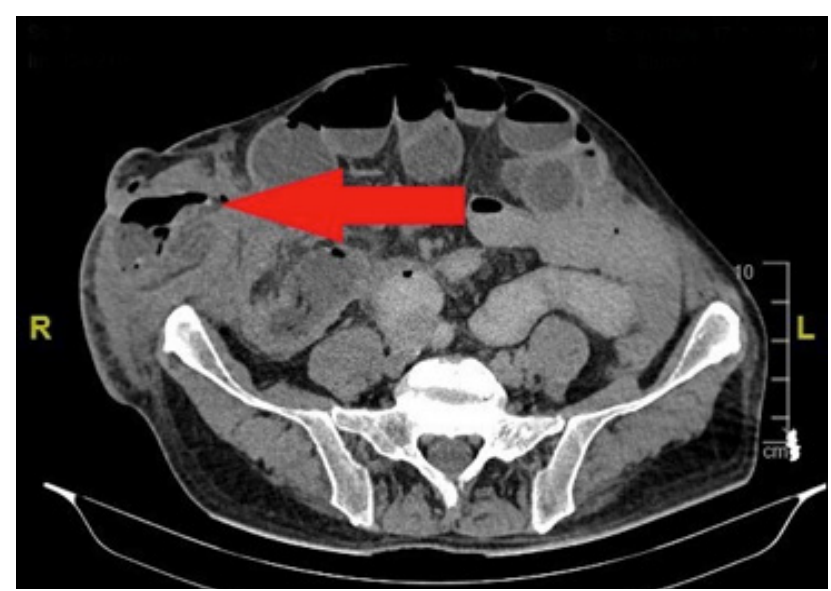

Figure 7. Comuputer Tomography imaging of perforation due to strangulated parastomal hernia
Beside general risk factors like age, high BMI, diabetes, respiratory diseases, and immunosuppression; the presence of other abdominal wall hernias, stoma extraction site, aperture size, and type of stoma are the other risk factors for parastomal hernia development [20]. Various studies showed different incidence rates depending on the type of stoma and follow up time. Generally, end colostomy has the highest parastomal hernia rate (\% 4-48). Loop colostomy and loop ileostomy have 0-30.8 percent and 0-6.2 percent parastomal herniation rates respectively [21].

Diagnosis can be made by clinical examination and computed tomography (CT) can confirm the diagnosis and can be helpful to detect small hernias which are not obvious in clinical examination. Intrastromal Ultrasonography is a relatively new imaging modality which can confirm parastomal hernia [22].

In order to prevent from a parastomal hernia which is nearly inevitable especially for end colostomies, prophylactic mesh usage during primary operation can be a safe and effective option [23-25]. The main controversy exists on the type of the mesh (biologic vs. composite) and the technique for mesh implementation (intraperitoneal, preperitoneal, retromuscular etc.) [25-28].

Conservative management can be possible for most of the patients who have a parastomal hernia but surgical intervention is necessary if the patient becomes symptomatic. The most convenient way is the reversal of the stoma if possible otherwise primary suture repair, stoma relocation and repair with prosthetic materials either by laparoscopic or open techniques are the treatment options. Local repair with primary closure of the fascia is a relatively simple procedure with low complication rates but recurrence rates are unacceptably high [29]. Although stoma relocation is superior to fascial repair it carries the risks of all stoma formation complications and risks of re-abdominal intrusion [30]. Both open and laparoscopic approaches described for parastoma hernia repair. Open techniques can be evaluated in terms of the type and the positioning of the mesh. High reluctance about prosthetic material used because of the fear of mesh infection, erosion and migration into bowel decreased by current evidence. Many studies which synthetic materials used for repair showed low mesh-related infection and recurrence rates [31,32]. Onlay, sublay or underlay (intraperitoneal) placement of prosthetic material is possible. All techniques have some advantages and disadvantages to each other (33). Onlay repair is consist of the reduction of hernia material, fascial repair and mesh placement above the fascia. Onlay technique has the advantage of avoidance from laparotomy. In retromuscular placement mesh placed, retromuscularly on the posterior rectus sheath. This can be performed either by laparotomy or peristomal stomal incision. The intraperitoneal repair can be performed either by laparoscopic or open fashioned. Two techniques (Sugarbaker, Keyhole) can be used. Sugarbaker technique has better outcomes in terms of recurrence if the laparoscopic approach preferred. Nevertheless, all techniques using 
prosthetic materials are superior from suture repair and can be applied with low morbidity. Open intra-peritoneal or sublay mesh placement has a slight superiority to other techniques in terms of recurrence [33].

\section{CONCLUSION}

Stoma creation is prone to complications. These complications can be life-threatening and effect the patients' quality of life. Preoperative preparation and postoperative close follow up are important for early diagnosis and treatment of complications. Using meticulous surgical technique, patient education and the presence of a consulting stoma therapist, preoperative stoma site marking are the important steps of good working stoma formation.

\section{CONFLICT Of INTEREST}

None.
[1] Malik T, Lee MJ, Harikrishnan AB. The incidence of stoma related morbidity - a systematic review of randomised controlled trials. Ann R Coll Surg Engl. 2018; 100: 501-08.

[2] Harris DA, Egbeare D, Jones S, et al. Complications and mortality following stoma formation. Annals of the Royal College of Surgeons of England. 2005; 87: 427-31.

[3] Nasvall P, Dahlstrand U, Lowenmark T, et al. Quality of life in patients with a permanent stoma after rectal cancer surgery. Quality of life research : an international journal of quality of life aspects of treatment, care and rehabilitation. 2017; 26: 55-64.

[4] Krouse R, Grant M, Ferrell B, et al. Quality of life outcomes in 599 cancer and non-cancer patients with colostomies. $J$ Surg Res. 2007; 138: 79-87.

[5] Shellito PC. Complications of abdominal stoma surgery. Diseases of the Colon \& Rectum. 1998; 41: 1562-72.

[6] Baykara ZG, Demir SG, Karadag A, et al. A multicenter, retrospective study to evaluate the effect of preoperative stoma site marking on stomal and peristomal complications. Ostomy/wound management. 2014; 60: 16-26.

[7] Park JJ, Del Pino A, Orsay CP, et al. Stoma complications: the Cook County Hospital experience. Dis Colon Rectum. 1999; 42: 1575-80.

[8] Kann BR. Early stomal complications. Clin Colon Rectal Surg. 2008; 21: 23-30.

[9] Parmar KL, Zammit M, Smith A, et al. A prospective audit of early stoma complications in colorectal cancer treatment throughout the Greater Manchester and Cheshire colorectal cancer network. Colorectal Dis. 2011; 13: 935-8.

[10] Bafford AC, Irani JL. Management and complications of stomas. Surg Clin North Am. 2013; 93: 145-66.

[11] Kiran RP, O'Brien-Ermlich B, Achkar JP, et al. Management of peristomal pyoderma gangrenosum. Dis Colon Rectum. 2005; 48: 1397-403.

[12] [Hughes AP, Jackson JM, Callen JP. Clinical Features and Treatment of Peristomal Pyoderma Gangrenosum. JAMA. 2000; 284: 1546-48.

[13] Shabbir J, Britton DC. Stoma complications: a literature overview. Colorectal Dis. 2010; 12: 958-64.

[14] Cottam J, Richards K, Hasted A, et al. Results of a nationwide prospective audit of stoma complications within 3 weeks of surgery. Colorectal Dis. 2007; 9: 834-8.
[15] Husain SG, Cataldo TE. Late stomal complications. Clin Colon Rectal Surg. 2008; 21: 31-40.

[16] Correa-Marinez A, Grenabo J, Bock D, et al. The type of stoma matters-morbidity in patients with obstructing colorectal cancer. International Journal of Colorectal Disease. 2018; 33: 1773-80.

[17] Davidson J, Healy C, Blackburn SC, et al. Laparoscopic Enteropexy for Prolapsing Stoma: A Case Series Describing a Novel Technique. J Laparoendosc Adv Surg Tech A. 2018; 28: 1135-38.

[18] Śmietański M, Szczepkowski M, Alexandre JA, et al. European Hernia Society classification of parastomal hernias. Hernia : the journal of hernias and abdominal wall surgery. 2014; 18: 1-6.

[19] Pearl RK. Parastomal hernias. World J Surg. 1989; 13: 569-72.

[20] Aquina CT, lannuzzi JC, Probst CP, et al. Parastomal Hernia: A Growing Problem with New Solutions. Digestive Surgery. 2014; 31: 366-76.

[21] Carne PWG, Robertson GM, Frizelle FA. Parastomal hernia. BJS. 2003; 90: 784-93.

[22] Gurmu A, Gunnarsson U, Strigård K. Imaging of parastomal hernia using three-dimensional intrastomal ultrasonography. BJS. 2011; 98: 1026-29.

[23] Wijeyekoon SP, Gurusamy K, El-Gendy K, et al. Prevention of Parastomal Herniation with Biologic/Composite Prosthetic Mesh: A Systematic Review and Meta-analysis of Randomized Controlled Trials. Journal of the American College of Surgeons. 2010; 211: 637-45.

[24] Jones HG, Rees M, Aboumarzouk OM, et al. Prosthetic mesh placement for the prevention of parastomal herniation. Cochrane Database Syst Rev. 2018; 7: Cd008905.

[25] Lambrecht JR, Larsen SG, Reiertsen O, et al. Prophylactic mesh at end-colostomy construction reduces parastomal hernia rate: a randomized trial. Colorectal Disease. 2015; 17: 0191-097.

[26] Gogenur I, Mortensen J, Harvald T, et al. Prevention of parastomal hernia by placement of a polypropylene mesh at the primary operation. Dis Colon Rectum. 2006; 49: 1131-5.

[27] Chapman SJ, Wood B, Drake TM, et al. Systematic Review and Meta-analysis of Prophylactic Mesh During Primary Stoma Formation to Prevent Parastomal Hernia. Diseases 
of the Colon \& Rectum. 2017; 60: 107-15.

[28] López-Cano M, Lozoya-Trujillo R, Quiroga S, et al. Use of a prosthetic mesh to prevent parastomal hernia during laparoscopic abdominoperineal resection: a randomized controlled trial. Hernia. 2012; 16: 661-67.

[29] Rieger N, Moore J, Hewett P, et al. Parastomal hernia repair. Colorectal Disease. 2004; 6: 203-05.

[30] Rubin MS, Schoetz DJ, Jr, Matthews JB. Parastomal Hernia: Is Stoma Relocation Superior to Fascial Repair? JAMA Surgery. 1994; 129: 413-19.
[31] Al Shakarchi J, Williams JG. Systematic review of open techniques for parastomal hernia repair. Techniques in coloproctology. 2014; 18: 427-32.

[32] Lüning TH, Spillenaar-Bilgen E-J. Parastomal hernia: complications of extra-peritoneal onlay mesh placement. Hernia. 2009; 13: 487.

[33] Hansson BME, Slater NJ, van der Velden AS, et al. Surgical Techniques for Parastomal Hernia Repair: A Systematic Review of the Literature. Annals of Surgery. 2012; 255: 685-95. 